\title{
OPEN Production of ethanol from Jerusalem artichoke by mycelial pellets
}

\author{
Chao Zhang ${ }^{1,2}$, Daoji Wu ${ }^{1,2^{*}}$, Hongqi Yang ${ }^{3}$ \& Huixue Ren ${ }^{1,2}$ \\ Mycelial pellets formed by Aspergillus niger A-15 were used to immobilize the ethanol producing yeast \\ Saccharomyces cerevisiae C-15. The operation parameters, such as agitation speed, temperature and \\ mixed proportion of strains were studied. The optimal adsorption $66.9 \%$ was obtained when speed \\ was $80 \mathrm{r} / \mathrm{min}$, temperature was $40^{\circ} \mathrm{C}$ and mixed proportion (mycelial pellets: yeasts) was $1: 10$. With \\ Jerusalem artichoke flour as substrate, $12.8 \%(\mathrm{~V} / \mathrm{V})$ of ethanol was obtained after $48 \mathrm{~h}$ by simultaneous \\ saccharification and fermentation using mycelial pellets. And mycelial pellets could tolerate $19 \%$ \\ (volume fraction) ethanol. The above results proved that this new technology was feasible, and it had \\ the advantages of higher ethanol yield, long service life, repeated use, easy operation and lower cost in \\ producing ethanol.
}

Corn is the major feedstock for the fuel ethanol production in China. However, this process consumes a large amount of corn, which poses a threat to national food security ${ }^{1-5}$. Jerusalem artichoke (JA) can be suitable for ethanol production as it contains $11-20 \%(\mathrm{w} / \mathrm{w})$ carbohydrate of which $70-90 \%$ is inulin. Inulin, a fructan-type polysaccharide, consists of $(2 \rightarrow 1)$ linked $\beta$-D-fructosyl residues $(n=2-60)$, usually with an $(1 \leftrightarrow 2) \alpha$-D-glucose end group. Inulin as feedstock for ethanol production is more advantageous than conventional material, such as starch ${ }^{6-8}$. For example, fermentable sugars can be obtained by enzymatic hydrolysis of inulin directly, which has the characteristics of simple process without high temperature liquefaction and low energy consumption. Compared with crop straw which is the representative of lignocellulosic biomass, ethanol production by inulin fermentation is easier to industrialize ${ }^{9-11}$. In addition, JA has the advantages of the high carbohydrate yields (5 and 14 ton/ha) and large-scale cultivation. This plant can be adapted to different agriculture conditions, because of the cold and drought tolerance, wind and sand resistance, saline tolerance, strong fecundity, and high pest and disease resistance ${ }^{12,13}$. Therefore, JA can be optimal non-grain feedstock for ethanol production. And there are many studies focused on producing ethanol using JA ${ }^{12-14}$.

Yeasts are frequently used in the process of ethanol production using JA as feedstock. However, yeast cannot secrete inulinase, and so inulin should be saccharified firstly. The most commonly used technology for the production of ethanol from JA is simultaneous saccharification and fermentation. Simultaneous saccharification and fermentation can convert the fermentable sugars from hydrolysate of inulin to ethanol simultaneously, preventing sugar accumulation which inhibits inulinase secretion, avoiding substrate inhibition, preventing bacteria pollution effectively, increasing ethanol yield. However, there are many shortcomings in this technology, such as lower ethanol yield and lower utilization rate of inulin ${ }^{15-19}$. In addition, there is a problem that strains cannot be reused, resulting in high production cost and environmental pollution. Cell immobilization technique can be used to solve the above problems. Immobilized yeasts can improve the efficiency of enzymatic reaction, so it has a good application prospect in the process of ethanol production. For example, Xue et al. ${ }^{20,21}$ reported that high-titer butanol production was observed with Clostridium acetobutylicum JB200 in a fibrous bed bioreactor. But the high cost of immobilization restricts its applications ${ }^{22-26}$. And most Saccharomyces cerevisiae can not directly utilize inulin, immobilization operation needs to fix a variety of strains, which will further increase the cost of immobilization. Furthermore, many investigations were focused on finding the high ethanol tolerance strains for ethanol production and relieving the inhibition of high concentration ethanol ${ }^{27-30}$.

Aiming at resolving the deficiency of the conventional technology, this study put forward a kind of carrier fermentation technology. At present, there are no reports of simultaneous saccharification and fermentation which are combined with immobilize carrier to producing ethanol. These two techniques are generally used separately, and

${ }^{1}$ School of Municipal and Environmental Engineering, Shandong Jianzhu University, JiNan, 250101, China. ${ }^{2}$ CoInnovation Center of Green Building, JiNan, 250101, China. ${ }^{3}$ School of foreign languages and literature, Nanjing Tech University, Nanjing, 210009, China. *email: 402187730@qq.com 


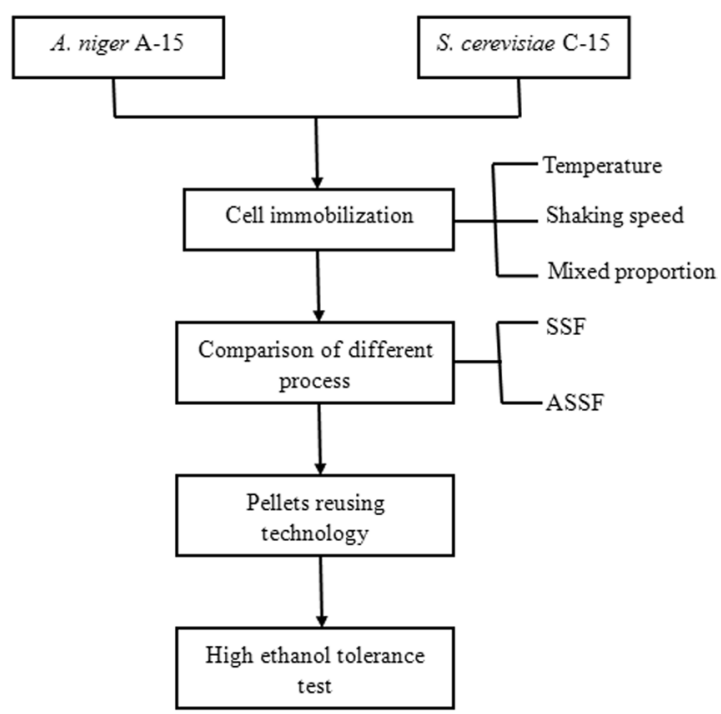

Figure 1. Flow chart of ASSF.

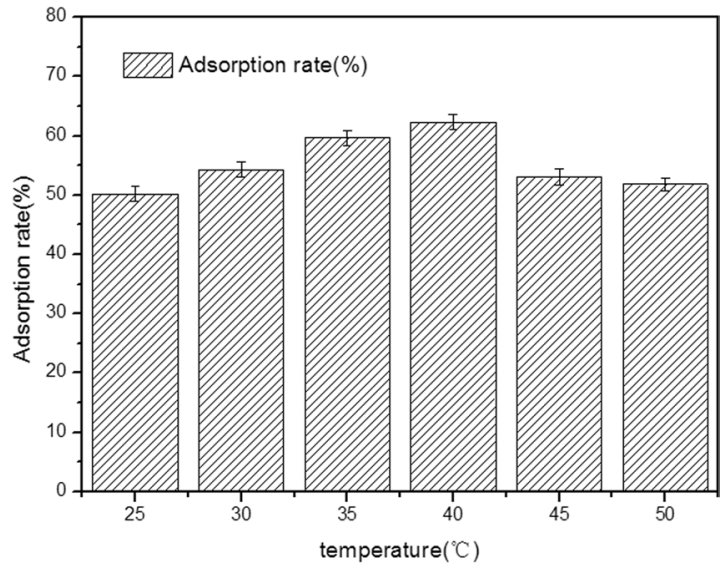

Figure 2. Effect of temperature on the adsorption rate of yeast.

there are no relevant reports about using immobilized pellets as fermenting microbe. This is the first time that this concept and technology are adopted to combine carrier with fermenting microbe in ethanol production. Because A. niger A-15 could secrete inulinase and form mycelial pellets, A. niger A-15 was used as carrier. As saccharification was required before fermentation, mycelial pellets formed by A. niger A-15 were used to immobilize the ethanol producing yeast $S$. cerevisiae $\mathrm{C}-15$. Ethanol was produced by mycelial pellets through simultaneous saccharification and fermentation, to simplify the process and improve the ethanol tolerance and ethanol yield of yeast.

\section{Results}

Effect of temperature on the adsorption rate of yeast. The process diagram of the whole experiment is shown in Fig. 1.To determine how different temperatures affected the adsorption rate of yeast, the pellets of $A$. niger A-15 were conducted in $500 \mathrm{ml}$ flask containing $100 \mathrm{~mL}$ yeast suspension. Then the yeast cells were adsorbed on a rotary shaker operating at different temperatures $\left(25,30,35,40,45,50^{\circ} \mathrm{C}\right)$ for $2 \mathrm{~h}$, shown in Fig. 2 . The maximum adsorption rate of yeast was about $62.3 \pm 0.1 \%$ at $40^{\circ} \mathrm{C}$. Therefore, temperature $40^{\circ} \mathrm{C}$ was employed in the following experiments.

Effect of shaking speed on the adsorption rate of yeast. To determine how different shaking speeds affected the adsorption rate of yeast, the pellets of $A$. niger A-15 were conducted in $500 \mathrm{~mL}$ flask containing $100 \mathrm{~mL}$ yeast suspension. Then the yeast cells were adsorbed on a rotary shaker operating at different speeds (40, $60,80,100,120 \mathrm{r} / \mathrm{min}$ ) for $2 \mathrm{~h}$, and the results are shown in Fig. 3. The maximum adsorption rate of yeast was about $65.1 \pm 0.1 \%$ at $80 \mathrm{r} / \mathrm{min}$. Therefore, the shaking speed $80 \mathrm{r} / \mathrm{min}$ was employed in the following experiments.

Effect of mixed proportion on the adsorption rate of yeast. The effect of mixed proportion mycelial pellets: yeasts $(1: 1,1: 3,1: 6,1: 10,1: 12)$ on the adsorption rate of yeast were investigated, and the results are shown in Fig. 4. The mixed proportion refers to the ratio between the number of mycelial pellets and yeast. 


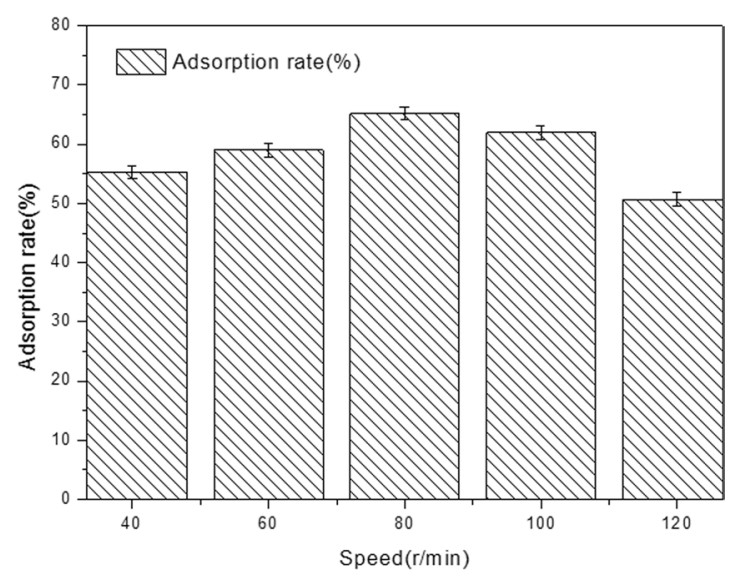

Figure 3. Effect of speed on the adsorption rate of yeast.

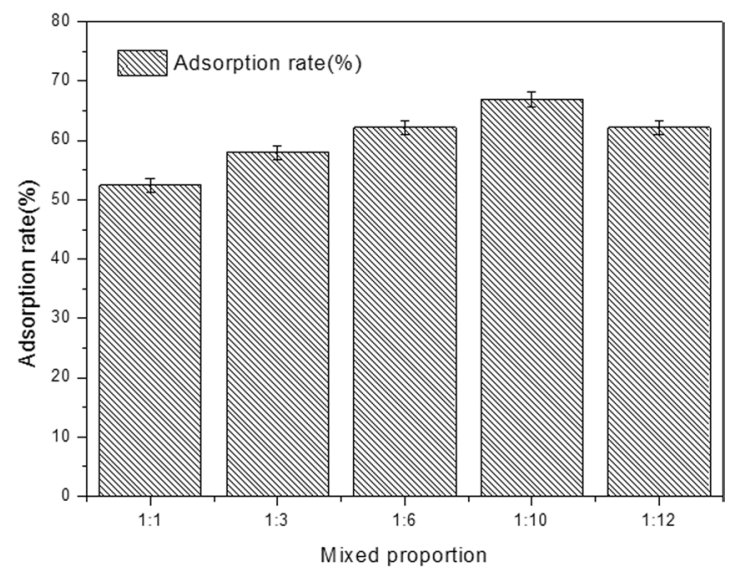

Figure 4. Effect of mixed proportion on the adsorption rate of yeast.

As shown in Fig. 4, the proportion of strains could affect the formation of mixed mycelial pellets. The adsorption rate of pellets increased with the concentration of yeast. The maximum adsorption rate of yeast was about $66.9 \pm 0.1 \%$ at $1: 10$. Any further increase in the concentration of yeast resulted in a decrease of the adsorption rate of pellets. This decrease-trend at high yeast concentration could be partly attributed to a result of no redundant adsorption sites on mycelial pellets.

In addition to the electrostatic adsorption of surface charges, mycelial pellets also secrete a kind of extracellular polymer film on the surface of mycelia, which is mainly composed of polysaccharides and proteins. It makes the surface of mycelial pellets have a greater adhesion force, and is more favorable for yeast adsorption than other biological carriers ${ }^{32}$. It can be seen from Fig. 5(a,b) that yeast is adsorbed to mycelia in large quantities. In addition, unlike the microporous structure of particulate activated carbon, the inner space of mycelial pellets is larger, which is conducive to the entry and adsorption of yeast.

Comparison of different fermentation modes. A bulk of literature has investigated the SSF process using Jerusalem artichoke as substrate for ethanol production ${ }^{16-19}$. However, as a new process, the feasibility and advantages of ASSF process were studied first in this study. Moreover, many processes in the literature use different strains, so it is impossible to compare these processes with ASSF under the same conditions. Therefore, this study chose a more representative process reported in literature 11 to compare with ASSF. Table 1 indicates the comparison of different fermentation modes. After $48 \mathrm{~h}$ cultivation, the ethanol yield by the pellets was approximately 1.19 times higher than that by conventional simultaneous saccharification and fermentation ( $12.8 \%$ versus $10.8 \%)$, and lower residual sugar concentration $(5.8 \mathrm{~g} / \mathrm{L}$ versus $1.5 \mathrm{~g} / \mathrm{L})$. Considering the ethanol yield and residual sugar concentration, carrier fermentation was much more economical. Moreover, the chemical oxygen demand (COD) of effluent showed that the COD of effluent produced after the ethanol distillation of the fermentation liquid was reduced from $54612 \mathrm{mg} / \mathrm{L}$ to $27641 \mathrm{mg} / \mathrm{L}$ of SSF, which is beneficial to reducing the pollution from the source.

In addition, due to the importance of inulinase in the fermentation process, the effect of ASSF process on inulinase production by $A$. niger was also investigated. Table 1 shows that ASSF process could also promote inulinase production by $A$. niger. The specific reason might be that yeasts and pellets bind more closely in local scope, which alleviated the substrate inhibition of inulinase to some extent. 

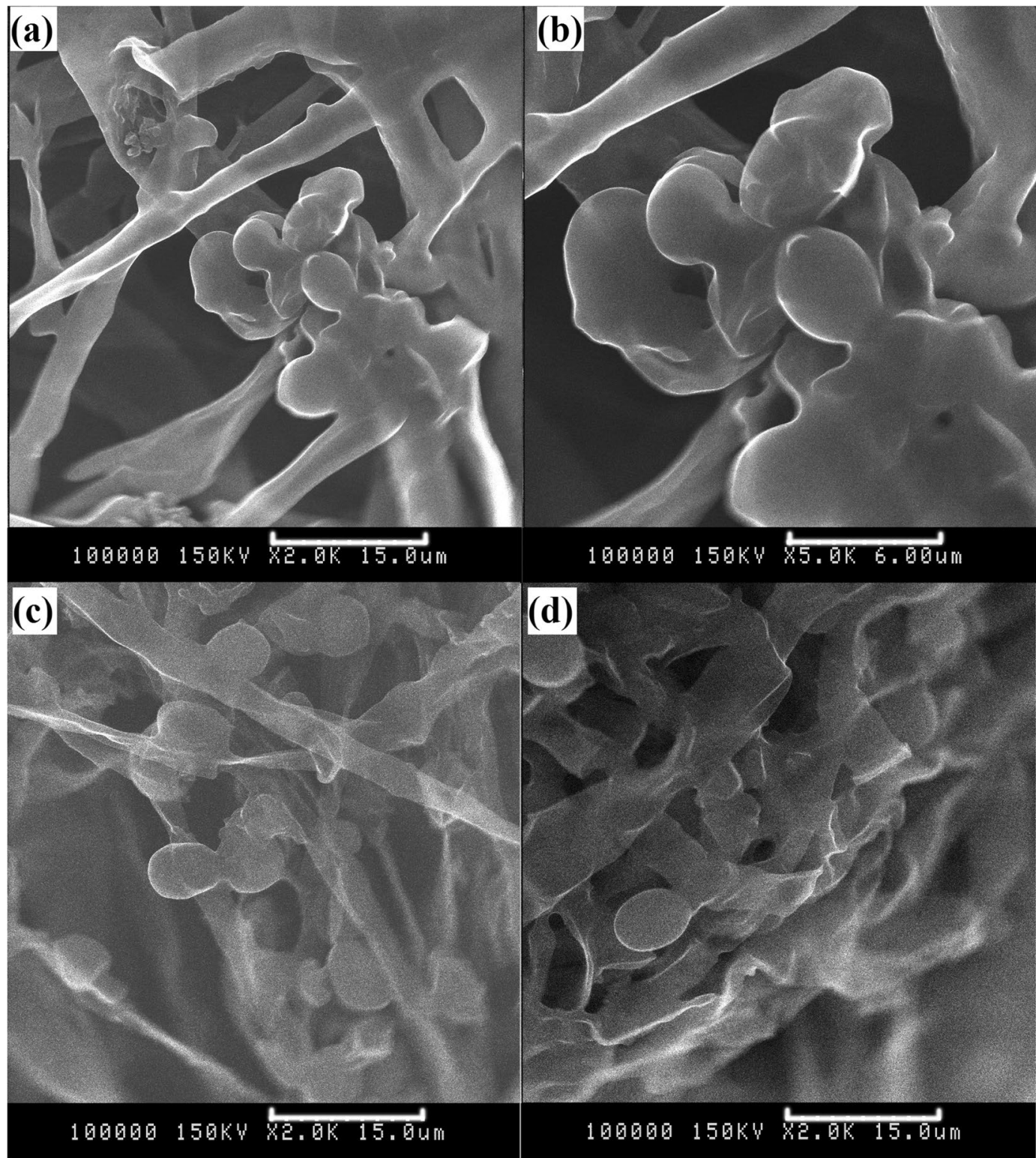

Figure 5. Adsorption of yeasts by mycelia pellets. (a,b) adsorption of yeasts by mycelia pellets (different magnification); (c) adsorption of yeasts by mycelia pellets (first recycle); (d) adsorption of yeasts by mycelia pellets (tenth recycle).

\begin{tabular}{|c|c|c|c|c|c|c|c|}
\hline Mode & $\begin{array}{l}\text { Ethanol } \\
\text { concentration(\%) }\end{array}$ & $\begin{array}{l}\text { Ethanol } \\
\text { yield(\%) }\end{array}$ & $\begin{array}{l}\text { Residual } \\
\text { sugar }(g / L)\end{array}$ & $\begin{array}{l}\text { Inulinase } \\
\text { activity }(\mathrm{U} / \mathrm{mL})\end{array}$ & $\begin{array}{l}\text { COD of effluent } \\
(\mathrm{mg} / \mathrm{L})\end{array}$ & Remarks & Reference \\
\hline $\mathrm{SSF}^{\mathrm{a}}$ & $10.82 \pm 0.37$ & $40.2 \pm 0.25$ & $5.8 \pm 0.11$ & $20.18 \pm 0.31$ & $54612 \pm 169$ & $\begin{array}{l}\text { Different strains, } \\
\text { same process }^{\mathrm{b}}\end{array}$ & 11 \\
\hline ASSF $^{a}$ & $12.81 \pm 0.25$ & $44.4 \pm 0.35$ & $1.5 \pm 0.17$ & $24.68 \pm 0.29$ & $27641 \pm 158$ & & This study \\
\hline
\end{tabular}

Table 1. Comparison of different fermentation modes. ${ }^{a}$ SSF: Conventional simultaneous saccharification and fermentation; ASSF: Adsorptive simultaneous saccharification and fermentation. ${ }^{b}$ Different strains, same process: Because the strains used in the reference could be not obtained in this study, we could only use the strains used in ASSF process, which was conducive to the comparison between different processes.

The stability of pellets reusing technology. The effect of recycle times on the fermentation were investigated, and the results are shown in Fig. 6. After recycling 10 times, the ethanol production remained stable at a high level. Figure 5(c,d) shows the SEM of mycelial pellets recycling 1 and 10 times. Meanwhile, mycelia pellets have good adsorptive stability of yeast. The ASSF has the advantages of long service life, repeated use, easy operation and low cost. Therefore, carrier fermentation had a good prospect of industrial production. 


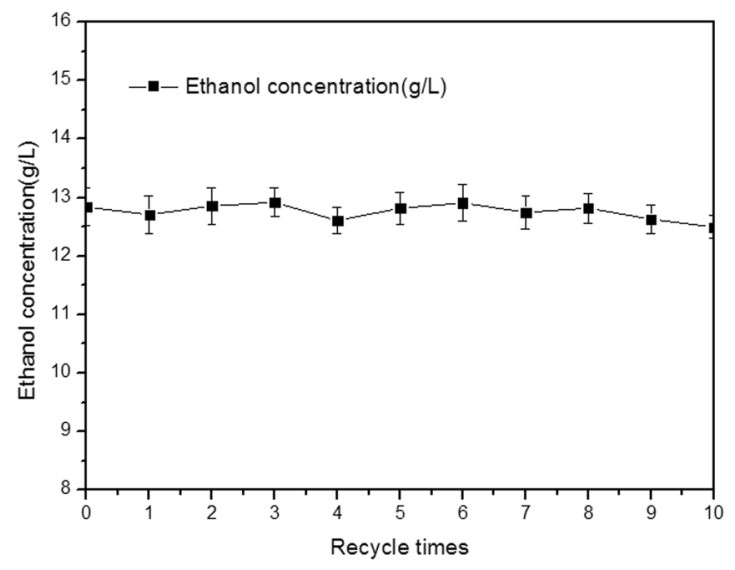

Figure 6. Effect of recycle times on the fermentation.

\begin{tabular}{|l|l|l|l|l|l|l|}
\hline \multirow{2}{*}{ Strain } & \multicolumn{6}{|l|}{ Ethanol concentration\% } \\
\cline { 2 - 8 } & $\mathbf{1 2}$ & $\mathbf{1 4}$ & $\mathbf{1 6}$ & $\mathbf{1 8}$ & $\mathbf{1 9}$ & $\mathbf{2 0}$ \\
\hline S.cerevisiae C-15 & +++ & + & - & - & - & - \\
\hline A. niger A-15 & +++ & ++ & - & - & - & - \\
\hline Mixed pellets & +++ & +++ & +++ & ++ & + & - \\
\hline
\end{tabular}

Table 2. Ethanol tolerance of the strains. ${ }^{*++}{ }^{++}$Indicates that the strains grow well; ${ }^{++}$indicates that the strains can grow; ${ }^{+}$indicates that some strains can grow; ${ }^{-}$indicates that the strains cannot grow.

High ethanol tolerance test. High concentration fermentation of ethanol is a hot research theme in recent years. The key technology of this study is to obtain yeast with high osmotic pressure and high ethanol yield. It has become a research hotspot to improve the ethanol tolerance of yeast and to relieve the inhibitory effect of high ethanol concentration. The results showed that the ethanol tolerance of yeast depended not only on the inherent tolerance ability of yeast cells to different concentrations of ethanol, but also on the close relationship between the plasma membrane lipid compositions of yeast, nutritional status, environmental conditions, and supplementary mode of carbohydrate substrate, etc $^{28}$. Critical ethanol concentration led to cleavage of plasma membrane phospholipids. With appropriate conditions of the plasma membrane of yeast, nutritional statusor environmental factors, the yeast can be resistant to ethanol toxicity. For example, with the temperature increasing, the phospholipid content of the plasma membrane in cell decreased rapidly to maintain the fluidity of the plasma membrane and cell viability ${ }^{30}$. Since $A$. niger is rich in lipoproteins which has been shown to improve the structure of the protoplasm membrane of yeast, the fermentation activity and ethanol tolerance of yeast are significantly improved. The combination of A. niger A-15 and S. cerevisiae C-15 could significantly improve the ethanol tolerance of $S$. cerevisiae C-15, as shown in Table 2.

As shown in Table 2, with the increase of ethanol volume fraction, the growth of A. niger $\mathrm{C}-15$ and S. cerevisiae A-15 was gradually inhibited. However, mixed pellets could enhance the ethanol tolerance of yeast and moulds, and mycelial pellets could tolerate 19\% (volume fraction) ethanol.

\section{Discussion}

This technology had the following advantages: (1) It could convert the fermentable sugars from hydrolysate of inulin to ethanol simultaneously, preventing sugar accumulation which inhibited inulinase secretion, avoiding substrate inhibition, preventing bacteria pollution effectively, increasing ethanol yield. (2) Because yeast could not directly utilize inulin, inulin was first decomposed by inulinase produced by $A$. niger, so the whole fermentation process was a mixed fermentation of yeast and $A$. niger. The immobilization of yeast with $A$. niger did not incur any immobilization cost. Therefore, this technology could reduce the high cost of cell immobilization. (3) Mycelial pellets could be easily recovered from broth, and the technology has the advantages of long service life, repeated use, simple operation, low cost and easy to industrialize. (4) It is generally known that mycelium of $A$. niger is rich in lipoprotein which can improve yeast viability and ethanol tolerance. Due to the microenvironment change of $S$. cerevisiae after immobilization by pellets, the tolerance of $S$. cerevisiae to ethanol was enhanced. (5) Using JA as feedstock for ethanol fermentation is more economical. Compared with lignocellulosic biomass, JA neither requires the pretreatment with high technical difficulty and high sugar consumption, nor does it need expensive enzyme. Compared with starch materials, due to the low degree of polymerization of inulin, the hydrolysis of JA is easier to achieve than that of starch which needs high temperature liquefaction, and synchronous inulinase hydrolysis can be obtained fermentable sugar, simple process flow, lower energy consumption. Therefore, JA can be a green and economical option for ethanol production.

Mycelial pellets formed by A. niger A-15 were used to immobilize S.cerevisiae C-15, the yeast producing ethanol. The operation parameters, such as agitation speed, temperature and mixed proportion of strains were 
studied. The optimal adsorption $66.9 \%$ was obtained when speed was $80 \mathrm{r} / \mathrm{min}$, temperature was $40{ }^{\circ} \mathrm{C}$ and mixed proportion was 1:10. With JA flour as substrate, $12.8 \%(\mathrm{~V} / \mathrm{V})$ of ethanol was obtained after $48 \mathrm{~h}$ by simultaneous saccharification and fermentation using mycelial pellets. Considering the ethanol yield and residual sugar concentration, carrier fermentation is much more economical in terms of time and energy. Moreover, mycelial pellets could tolerate $19 \%$ (volume fraction) ethanol. The above results indicate that this new technology is feasible in producing ethanol.

In addition, this study mainly investigated the feasibility and advantages of ASSF as a new technology. Development of strains with high alcohol tolerance is a research hotspot in ethanol industry. More detailed examinations like AFM (Atomic Force Microscope) will be used to explain the improved ethanol tolerance of pellets in future research.

\section{Materials and Methods}

Microorganism and culture medium. The A. niger A-15 (CMCC98003) was stored in our laboratory. The S. cerevisiae C-15 was purchased from ANGEL YEAST CO., LTD.

Jerusalem artichoke was obtained from the local market in JiNan, China. The preparation of JA powder was to cut fresh JA into thin slices and air-dry them at $60^{\circ} \mathrm{C}$ for $48 \mathrm{~h}$, so as to reduce the water content to about $5 \%$, and then ground to 60 mesh.

Seed medium(SM), in $\mathrm{g} / \mathrm{L}$ : yeast extract, 10; peptone, 20; glucose, 20. The $\mathrm{pH}$ was adjusted to 7.0 by $\mathrm{HCl}$ or $\mathrm{NaOH}$.

Medium for enzyme production (ME), in g/L: JA powder, 25; $\left(\mathrm{NH}_{4}\right)_{2} \mathrm{SO}_{4}$, 5; peptone, $10 ; \mathrm{KH}_{2} \mathrm{PO}_{4}, 6 ; \mathrm{NaCl}, 5$; $\mathrm{MgSO}_{4} \cdot 7 \mathrm{H}_{2} \mathrm{O}, 0.5 ; \mathrm{FeSO}_{4} \cdot 7 \mathrm{H}_{2} \mathrm{O}, 0.001$. The $\mathrm{pH}$ was adjusted to 5.0 by $\mathrm{HCl}$ or $\mathrm{NaOH}$.

Fermentation medium (FM), in g/L: JA powder, 200; peptone, 5; yeast extract, 5; $\left(\mathrm{NH}_{4}\right)_{2} \mathrm{SO}_{4}, 2 ; \mathrm{KH}_{2} \mathrm{PO}_{4} 2$; corn syrup, 5 . The $\mathrm{pH}$ was adjusted to 5.0 by $\mathrm{HCl}$ or $\mathrm{NaOH}$.

YPD solid medium, in g/L: yeast extract, 10; peptone, 20; glucose, 20; agar, 20. Natural pH.

These media were autoclaved at $121^{\circ} \mathrm{C}$ for $20 \mathrm{~min}$.

Cell culture. The culture of $S$. cerevisiae C-15 was harvested in $250 \mathrm{~mL}$ flask containing $100 \mathrm{~mL}$ seed medium with $5 \%$ of inoculum. The flask cultivated on a rotary shaker operating at $30^{\circ} \mathrm{C}$ and $100 \mathrm{r} / \mathrm{min}$ for $30 \mathrm{~h}$. The broth was centrifuged at $5000 \mathrm{r} / \mathrm{min}$ for $5 \mathrm{~min}$, and then the cells were collected. Three replicates were carried out for each experiment.

A. niger A-15 was mixed with sterile water to form spore suspensions. The culture of mycelial pellets was grown in $250 \mathrm{ml}$ flask containing $100 \mathrm{ml} \mathrm{ME}$ medium with $4 \%$ of inoculum. The flask was cultivated on a rotary shaker operating at $30^{\circ} \mathrm{C}$ and $40 \mathrm{r} / \mathrm{min}$ for $30 \mathrm{~h}$. The broth was centrifuged at $5000 \mathrm{r} / \mathrm{min}$ for $5 \mathrm{~min}$, and mycelial pellets formed by $A$. niger A-15 were collected by filtration.

Adsorption test. Yeast cells were suspended with $100 \mathrm{~mL}$ distilled water in a $250 \mathrm{~mL}$ Erlenmeyer flask, into which $5 \mathrm{~g}$ (wet weight) of pellet was added. The yeast cells were adsorbed at different temperatures, shaking speeds and different strains of the mixing ratio for $2 \mathrm{~h}$. Then the cells were filtered by two-layer gauze, determined of the concentration of the cells that remained in the filtrate, and the adsorption rate was calculated with spectrophotometry.

$$
\begin{aligned}
\text { Adsorption rate }(R)= & \left(\mathrm{OD}_{600} \text { of mixture before adsorption }-\mathrm{OD}_{600} \text { of mixture after adsorption }\right) \\
& / \mathrm{OD}_{600} \text { of mixture before adsorption } \times 100 \%
\end{aligned}
$$

Conventional simultaneous saccharification and fermentation. Yeast cells and A. niger Spore suspension were collected as described earlier, then mixed them in a certain proportion. The mixture of cells was grown in $500 \mathrm{~mL}$ flask containing $200 \mathrm{ml} \mathrm{FM}$ with $10 \%$ of inoculum(volume fraction). The flask was cultivated on a rotary shaker operating at $30^{\circ} \mathrm{C}$ and $40 \mathrm{r} / \mathrm{min}$ for $48 \mathrm{~h}$.

Adsorptive simultaneous saccharification and fermentation. Yeast cells and A. niger spore suspension were collected as described earlier, then adsorbed in optimal temperature, agitation speed and mixed proportion of strains for $2 \mathrm{~h}$. The mixture of cells was conducted in $500 \mathrm{~mL}$ flask containing $200 \mathrm{ml}$ fermentation medium with $10 \%$ of inoculum(volume fraction). The flask was cultivated to produce ethanol on a rotary shaker operating at $30^{\circ} \mathrm{C}$ and $40 \mathrm{r} / \mathrm{min}$ for $48 \mathrm{~h}$.

The stability of pellets reusing technology. Broth was centrifuged at $1000 \mathrm{r} / \mathrm{min}$ for $15 \mathrm{~min}$, the collected mycelial cells were washed with normal saline and centrifuged at $1000 \mathrm{r} / \mathrm{min}$ for $5 \mathrm{~min}$, and all mycelial pellets were transferred into fresh fermentation medium.

High ethanol tolerance test. To ensure the ethanol tolerance of the mycelial pellets, these pellets were cultured in YPD solid medium supplemented with various concentrations of ethanol (12\%, 14\%, 16\%, 18\%, 19\% and $20 \%)$. Pellets were inoculated at an initial cell density of $2 \times 10^{8}$ cells $/ \mathrm{ml}$. Samples were collected, diluted, and plated on YPD solid medium. After incubation at $37^{\circ} \mathrm{C}$ for 2 days, the colonies appearing on the plates were counted. Viability was expressed as a percentage of colony-forming units of the high ethanol treatment compared with control for each culture of the strains. Compared with the control group, the survival rate of strains was between $50 \%$ and $100 \%$, which meant that the strain grew well. Compared with the control group, the survival rate of strains between $50 \%$ and $100 \%$ indicates that the strains grew well, the survival rate between $20 \%$ and $50 \%$ means that the strains can grow, while the survival rate between $0 \%$ and $20 \%$ means that the strains can not grow. The survival rate of strains was between $0 \%$ and $20 \%$, which meant that it was difficult for the strain to grow. 
Analytical methods. The number of yeasts and fungi was determined by plate counting. al. ${ }^{17}$.

Ethanol concentration in the fermented medium was determined according to the methods given by $\mathrm{Ge} e t$

Residual sugars were determined by the 3,5-dinitrosalicylic acid (DNS) method ${ }^{31}$. Total sugar was measured following the method reported previously ${ }^{31}$. Sample solution $(1 \mathrm{~mL}), 50 \mathrm{ml}$ of $2.8 \% \mathrm{H}_{2} \mathrm{SO}_{4}$ was mixed and then heated at $100^{\circ} \mathrm{C}$ to hydrolyze the carbohydrates. After $1 \mathrm{~h}$, the solution was neutralized with $5 \mathrm{~mol} / \mathrm{L} \mathrm{NaOH}$ solution, and fixed capacity to $100 \mathrm{~mL}$. The reducing sugar in the solution was determined by DNS method.

Inulinase activity was determined according to the method described by Susana $\mathrm{M}^{17}$. One unit of inulinase activity $(\mathrm{U} / \mathrm{mL})$ was defined as the amount of enzyme responsible for the production of $1 \mu \mathrm{mol}$ of reducing sugar per minute at $55^{\circ} \mathrm{C}$ and $\mathrm{pH} 5.4$.

$$
\text { Inulinase activity }=\rho(\text { glucose }) \times \text { dilution rate } \times 1000 /(180 \times 10)
$$

where 180 is the molecular weight of glucose $(\mathrm{mg} / \mathrm{L}), 10$ is the reaction time $(\mathrm{min}), 1000$ is the unit conversion factor.

Scanning electron microscopy (SEM) was used to observe the structure and adsorption of mycelial pellets, and specific operation steps were determined according to the literature ${ }^{32}$.

$$
\text { The yield of ethanol }=\mathrm{m}(\text { Ethanol production }) / m \text { (the decrease of total sugar in broth) }
$$

Chemical oxygen demand (COD) was determined by potassium dichromate method ${ }^{31}$.

Received: 12 June 2019; Accepted: 25 November 2019;

Published online: 06 December 2019

\section{References}

1. Li, Y. J. et al. Engineered yeast with a CO2-fixation pathway to improve the bio-ethanol production from xylose-mixed sugars. Scientific Reports 7, 43875 (2017).

2. Li, K., Qin, J. C., Liu, C. G. \& Bai, F. W. Optimization of pretreatment, enzymatic hydrolysis and fermentation for more efficient ethanol production by Jerusalem artichoke stalk. Bioresour Technol 221, 188-194 (2016).

3. Khatun, M. M., Liu, C. G., Zhao, X. Q., Yuan, W. J. \& Bai, F. W. Consolidated ethanol production from Jerusalem artichoke tubers at elevated temperature by Saccharomyces cerevisiae engineered with inulinase expression through cell surface display. Journal of Industrial Microbiology \& Biotechnology 44, 295-301 (2017).

4. Tanimura, A. et al. Direct ethanol production from starch using a natural isolate, Scheffersomyces shehatae: Toward consolidated bioprocessing. Scientific Reports 5, 9593 (2015).

5. Matías, J., Encinar, J. M., González, J. \& González, J. F. Optimisation of ethanol fermentation of Jerusalem artichoke tuber juice using simple technology for a decentralised and sustainable ethanol production. Energy for Sustainable Development 25, 34-39 (2015).

6. Mathew, A. S., Wang, J., Luo, J. \& Yau, S. T. Enhanced ethanol production via electrostatically accelerated fermentation of glucose using Saccharomyces cerevisiae. Scientific Reports 5, 15713 (2015).

7. Liu, C. G., Hao, X. M., Lin, Y. H. \& Bai, F. W. Corrigendum: Redox potential driven aeration during very-high-gravity ethanol fermentation by using flocculating yeast. Scientific Reports 6, 25763 (2016).

8. Du, Y. C., Long, X. H., Liu, Z. P. \& Shao, H. B. Optimizing medium for producing ethanol from industrial crop Jerusalem artichoke by one-step fermentation and recombinant Saccharomyces cerevisiae. Giornale Botanico Italiano 148, 118-126 (2014).

9. Li, Y., Liu, G. L. \& Chi, Z. M. Ethanol production from inulin and unsterilized meal of Jerusalem artichoke tubers by Saccharomyces sp. W0 expressing the endo-inulinase gene from Arthrobacter sp. Bioresource Technology 147, 254 (2013).

10. Guo, L., Jian, Z., Hu, F., Ryu, D. D. \& Jie, B. Consolidated bioprocessing of highly concentrated jerusalem artichoke tubers for simultaneous saccharification and ethanol fermentation. Biotechnology \& Bioengineering 110, 2606-2615 (2013).

11. Dahiya, P., Bhat, D. S. \& Thakur, J. K. Expression of AtMed15 of Arabidopsis in yeast causes flocculation and increases ethanol production in yeast culture. Scientific Reports 6, 27967 (2016).

12. Santos Dos, L. V. et al. Unraveling the genetic basis of xylose consumption in engineeredSaccharomyces cerevisiaestrains. Scientific Reports 6, 38676 (2016).

13. Kim, S., Park, J. M. \& Kim, C. H. Ethanol production using whole plant biomass of Jerusalem artichoke by Kluyveromyces marxianus CBS1555. Applied Biochemistry \&. Biotechnology 169, 1531 (2013).

14. Choi, E. S., Lee, H. \& Jeon, J. H. Direct ethanol fermentation from Jerusalem artichoke using Saccharomyces cerevisiae KCCM50549 Without inulinase-pretreatment. Journal of Biotechnology 150, 154-154 (2010).

15. Unrean, P. \& Khajeeram, S. Optimization and techno-economic assessment of high-solid fed-batch saccharification and ethanol fermentation by Scheffersomyces stipitis and Saccharomyces cerevisiae consortium. Renewable Energy 99, 1062-1072 (2016).

16. Chang, B., Yuan, W., Zhao, X. \& Bai, F. Ethanol production from Jerusalem artichoke juice using self-flocculating yeast. Journal of Biotechnology 136, S272-S272 (2008).

17. Ge, X. Y. \& Zhang, W. G. A shortcut to the production of high ethanol concentration from Jerusalem artichoke tubers. Food Technology \&. Biotechnology 43, 241-246 (2005).

18. Szambelan, K., Nowak, J. \& Czarnecki, Z. Use of Zymomonas mobilis and Saccharomyces cerevisiae mixed with Kluyveromyces fragilis for improved ethanol production from Jerusalem artichoke tubers. Biotechnology Letters 26, 845-848 (2004).

19. Lin, Y. \& Tanaka, S. Ethanol fermentation from biomass resources: current state and prospects. Applied Microbiology \& Biotechnology 69, 627 (2006).

20. Xue, C. et al. Two-stage in situ gas stripping for enhanced butanol fermentation and energy-saving product recovery. Bioresource Technology 135, 396-402, https://doi.org/10.1016/j.biortech.2012.07.062 (2013).

21. Xue, C. et al. A novel in situ gas stripping-pervaporation process integrated with acetone-butanol-ethanol fermentation for hyper n-butanol production. Biotechnology and Bioengineering 113, 120-129, https://doi.org/10.1002/bit.25666 (2016).

22. Steele, B., Raj, S., Nghiem, J. \& Stowers, M. Enzyme recovery and recycling following hydrolysis of ammonia fiber explosion-treated corn stover. Applied Biochemistry \& Biotechnology Part A Enzyme Engineering \&. Biotechnology 124, 901-910 (2005).

23. Charoensopharat, K., Thanonkeo, P., Thanonkeo, S. \& Yamada, M. Ethanol production from Jerusalem artichoke tubers at high temperature by newly isolated thermotolerant inulin-utilizing yeast Kluyveromyces marxianus using consolidated bioprocessing. Antonie Van Leeuwenhoek 108, 173 (2015).

24. Shahbazi, A., Li, Y. \& Mims, M. R. Application of sequential aqueous steam treatments to the fractionation of softwood. Applied Biochemistry \& Biotechnology Part A Enzyme Engineering \&. Biotechnology 121-124, 973 (2005). 
25. Schell, D. J., Farmer, J., Newman, M. \& Mcmillan, J. D. Dilute-sulfuric acid pretreatment of corn stover in pilot-scale reactor. (Humana Press, 2003).

26. Rodriguezchong, A., Ramirez, J. A., Garrote, G. \& Vazquez, M. Hydrolysis of sugar cane bagasse using nitric acid: a kinetic assessment. Journal of Food Engineering 61, 143-152 (2004).

27. Kang, S. W., Park, Y. S., Lee, J. S., Hong, S. I. \& Kim, S. W. Production of cellulases and hemicellulases by Aspergillus niger KK2 from lignocellulosic biomass. Bioresource Technology 91, 153-156 (2004).

28. Aden, A. et al. Lignocellulosic Biomass to Ethanol Process Design and Economics Utilizing Co-Current Dilute Acid Prehydrolysis and Enzymatic Hydrolysis for Corn Stover (2002).

29. Ohta, K., Hamada, S. \& Nakamura, T. Production of high concentrations of ethanol from inulin by simultaneous saccharification and fermentation using Aspergillus niger and Saccharomyces cerevisiae. Applied \&. Environmental Microbiology 59, 729-733 (1993).

30. Hayashida, S. \& Ohta, K. Formation of high concentrations of alcohol by various yeasts. Journal of the Institute of Brewing $87,42-44$ (2013).

31. Gilcreas, F. W. Standard methods for the examination of water and waste water. (APHA, 1971).

32. Higashiyama, K., Murakami, K., Tsujimura, H., Matsumoto, N. \& Fujikawa, S. Effects of dissolved oxygen on the morphology of an arachidonic acid production by Mortierella alpina 1S-4. Biotechnology and Bioengineering 63, 442-448 (1999).

\section{Acknowledgements}

This research was financially supported by State Key Laboratory of Microbial Technology (M2012-14), Shandong University.

\section{Author contributions}

C.Z. and D.J.W. conceived of the study. C.Z. designed experiments, analyzed data, and performed experiments with assistance from H.X.R., C.Z. and H.Q.Y drafted the manuscript. All authors read and approved the final manuscript.

\section{Competing interests}

The authors declare no competing interests.

\section{Additional information}

Correspondence and requests for materials should be addressed to D.W.

Reprints and permissions information is available at www.nature.com/reprints.

Publisher's note Springer Nature remains neutral with regard to jurisdictional claims in published maps and institutional affiliations.

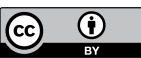

Open Access This article is licensed under a Creative Commons Attribution 4.0 International License, which permits use, sharing, adaptation, distribution and reproduction in any medium or format, as long as you give appropriate credit to the original author(s) and the source, provide a link to the Creative Commons license, and indicate if changes were made. The images or other third party material in this article are included in the article's Creative Commons license, unless indicated otherwise in a credit line to the material. If material is not included in the article's Creative Commons license and your intended use is not permitted by statutory regulation or exceeds the permitted use, you will need to obtain permission directly from the copyright holder. To view a copy of this license, visit http://creativecommons.org/licenses/by/4.0/.

(C) The Author(s) 2019 